Research Article

\title{
Exosomes Derived from Brain Metastatic Breast Cancer Cells Destroy the Blood-Brain Barrier by Carrying lncRNA GS1-600G8.5
}

\author{
Yunhe Lu $\mathbb{D}$, ${ }^{1,2}$ Lei Chen $\mathbb{D}$, ${ }^{1,2}$ Liangdong $L i \mathbb{D}^{1,2}$ and Yiqun Cao $\left.\mathbb{D}\right)^{1,2}$ \\ ${ }^{1}$ Department of Neurosurgery, Fudan University Shanghai Cancer Center, Shanghai 200032, China \\ ${ }^{2}$ Department of Oncology, Shanghai Medical College, Fudan University, Shanghai 200032, China \\ Correspondence should be addressed to Yunhe Lu; luyunhe2017@163.com and Yiqun Cao; yiqun_cao@126.com
}

Received 18 December 2019; Accepted 11 March 2020; Published 6 April 2020

Academic Editor: Taiyoun Rhim

Copyright (c) 2020 Yunhe Lu et al. This is an open access article distributed under the Creative Commons Attribution License, which permits unrestricted use, distribution, and reproduction in any medium, provided the original work is properly cited.

\begin{abstract}
Brain metastasis is a major cause of death in breast cancer patients. The greatest event for brain metastasis is the breaching of the blood-brain barrier (BBB) by cancer cells. The role of exosomes in cancer metastasis is clear, whereas the role of exosomes in the integrity of the $\mathrm{BBB}$ is unknown. Here, we established a highly brain metastatic breast cancer cell line by three cycles of in vivo selection. The effect of exosomes on the $\mathrm{BBB}$ was evaluated in vitro by tracking, transepithelial/transendothelial electrical resistance (TEER), and permeability assays. BBB-associated exosomal long noncoding RNA (lncRNA) was selected from the GEO dataset and verified by real-time PCR, TEER, permeability, and Transwell assays. The cells obtained by the in vivo selection showed higher brain metastatic capacity in vivo and higher migration and invasion in vitro compared to the parental cells. Exosomes from the highly brain metastatic cells were internalized by brain microvascular endothelial cells (BMECs), which reduced TEER and increased permeability of BBB. The exosomes derived from the highly metastatic cells promoted invasion of the breast cancer cells in the BBB model. IncRNA GS1-600G8.5 was highly expressed in the highly brain metastatic cells and their exosomes, as compared to the samples with reduced metastatic behavior. Silencing of GS1-600G8.5 significantly abrogated the BBB destructive effect of exosomes. GS1-600G8.5-deficient exosomes failed to promote the infiltration of cancer cells through the BBB. Furthermore, BMECs treated with GS1-600G8.5-deprived exosomes expressed higher tight junction proteins than those treated with the control exosomes. These data suggest the exosomes derived from highly brain metastatic breast cancer cells might destroy the BBB system and promote the passage of cancer cells across the BBB, by transferring lncRNA GS1600G8.5.
\end{abstract}

\section{Introduction}

Breast cancer is the most common cancer in women worldwide and is the second leading cause of cancer death among females [1]. Metastasis is the leading cause of morbidity and mortality in breast cancer patients. Brain metastasis occurs in approximately 15 to $30 \%$ of breast cancer patients, with the highest incidence in breast cancer patients with trinegative or basal tumors and Her-2 positive tumors [2, 3]. Although promising advances have recently been made in the treatment of breast cancer, the prognosis of breast cancer patients with brain metastasis remains poor. Therefore, novel insights into the process of brain metastasis in breast cancer are urgently needed.

A vital event in the migration of cancer cells to the brain is the crossing of the blood-brain barrier (BBB). The BBB is comprised of brain microvascular endothelial cells (BMECs), pericytes, astrocytes, endothelial basement membranes, and adjacent neurons [4]. However, how the cancer cells across the $\mathrm{BBB}$ to cause brain metastasis is unclear.

Long noncoding RNAs (lncRNAs) are a major focus in cancer research, reflecting their diverse mechanisms in the occurrence and progression of cancer [5]. lncRNAs are at least 200 nucleotides in length and do not encode proteins. 
Nonetheless, they are vital in various biological processes, including cell differentiation, intracellular homeostasis, genomic imprinting and organogenesis, and various pathological processes [6]. IncRNAs are also important during the metastasis of cancer. For instance, lncRNA AFAP1-AS1 promotes metastasis of nasopharyngeal carcinoma by sponging microRNA- (miR-) 423-5p and regulating the Rho/Rac signaling pathway [7]. MALAT1 lncRNA suppresses lung metastasis of breast cancer cells by binding and inactivating the prometastatic transcriptional enhanced associate domain (TEAD) transcription factor and preventing the binding of TEAD to the target gene promoters [8]. Noncoding RNA activated by DNA damage (NORAD) IncRNA suppresses breast cancer metastasis by sequestering S100P [9]. Moreover, lnc-BM promotes brain metastasis of breast cancer by enhancing the adhesion of cancer cells to cerebral vascular endothelial cells by the JAK2/STAT3 pathway [10]. However, the roles of lncRNA in brain metastasis of breast cancer are still largely unknown.

Exosomes are extracellular vesicles 30 to $100 \mathrm{~nm}$ in diameter, which are released into the extracellular environment by different types of cells [11]. A variety of molecules, including miRNAs, mRNAs, and lncRNAs, can be carried within exosomes and transferred from donor cells to recipient cells to trigger phenotypic changes in the tumor microenvironment. Exosomes appear to be important in cancer development, including the formation of premetastatic niches. Exosomes secreted by highly metastatic melanoma cells promote metastatic behavior of the primary tumor by inducing vascular leakage at the premetastatic site and reprogramming bone marrow progenitor cells to a vasogenic phenotype [12]. Exosomal miRNA secreted by hepatocellular carcinoma cells can increase vascular permeability by targeting endothelial connexin to promote tumor lung metastasis [13]. Astrocytederived exosomal miR-19a promotes brain metastasis of breast cancer cells by reversibly downregulating the expression of phosphatase and tensin homolog (PTEN) in cancer cells and increasing CCL2 secretion and myeloid recruitment [14]. However, whether exosomal lncRNAs are also involved in the brain metastasis of breast cancer is unknown.

In this study, we aimed to investigate the roles of exosomal lncRNAs in the brain metastasis of breast cancer. A brain metastasis breast cancer cell line was established by injecting immunodeficient female mice with MDA-MB-231-lucD3H2LN cells, which are highly tumorigenic and metastatic. The effect of exosomal lncRNA on the ability of the cells to cross the $\mathrm{BBB}$ was evaluated using an in vitro model of $\mathrm{BBB}$.

\section{Materials and Methods}

2.1. Establishment of Brain Metastatic Cells. The animal experiments were performed in strict accordance with the Guide for the Care and Use of Laboratory Animals of the National Institutes of Health. This study was approved by the Institutional Animal Care and Use Committee of the Fudan University Shanghai Cancer Center. The brain metastatic cells were established according to a previous study [15]. Female wild-type BALB/c mice with 6 to 8 weeks of age were obtained from Shanghai Ruitamos Biotechnology
Co. LTD. (Shanghai, China). A cell suspension containing 2 $\times 10^{5}$ MDA-MB-231-luc-D3H2LN breast cancer cells (MDA231) in a volume of $100 \mu \mathrm{l}$ was injected into the left cardiac ventricle of anesthetized mice. The metastasis of breast tumor cells was monitored by weekly bioluminescence imaging using an IVIS Spectrum device (Caliper Life Science, Hopkinton, MA, USA). After 30 days, brain metastatic tumor cells were dissociated and grown in culture, the generated cell group (brain metastatic cell group 1, MDABR1) was selected in the second and third round in vivo selection, yielding MDABR3 cell populations that displayed significantly increased brain metastasis.

2.2. Cell Culture. MDA-MB-231-luc-D3H1 cells (Xenogen Co., Alameda, CA) and the obtained MDABR3 cells were cultured in RPMI1640 medium and supplemented with $10 \%$ heat-inactivated fetal bovine serum (FBS, Invitrogen, Carlsbad, CA, USA) and antibiotic-antimycotic agents. Human astrocytes (Procell, Wuhan, China) were cultured in DMEM medium containing 10\% FBS (Invitrogen) and antibiotic-antimycotic agents. Human pericytes and brain capillary epithelial cells (BMECs, Procell) were cultured in M199 medium containing $10 \% \mathrm{FBS}$ at $37^{\circ} \mathrm{C}$ in an atmosphere of $5 \% \mathrm{CO}_{2}$.

2.3. Isolation of Exosomes. Cells were grown to logarithmic phase, and the medium was replaced with a serum-free medium. Culture was continued for $48 \mathrm{~h}$. The culture was centrifuge at $300 \mathrm{~g}$ for $10 \mathrm{~min}$. The supernatant was collected and dead cells were removed by centrifugation at $2000 \times g$ for $10 \mathrm{~min}$, followed by centrifugation at $10000 \times g$ for $30 \mathrm{~min}$ to remove cell debris. The supernatant was ultracentrifuged at $4^{\circ} \mathrm{C}, 100000 \times g$ for $1 \mathrm{~h}$. This step was repeated. The supernatant was removed, and the exosome precipitate was added to precooled $1 \times$ phosphate-buffered saline (PBS).

2.4. Transmission Electron Microscopy (TEM). Exosomes were incubated on a carbon-coated copper grid, for $5 \mathrm{~min}$ and stained by applying a drop of $2 \%$ phosphotungstic acid on the grid for $3 \mathrm{~min}$. Absorbing paper was used to remove the excess liquid, and the grid was air dried for $15 \mathrm{~min}$. The exosomes were observed by TEM.

2.5. Transwell Assay. The Transwell assay was used to evaluate cell migration and invasion. Cell migration was assessed using $0.8 \mu \mathrm{m}$ 24-well chambers (353097, Falcon), and cell invasion was performed using BioCoat ${ }^{\mathrm{TM}}$ Matrigel $^{\circledR} 0.8 \mu \mathrm{m}$ 24-well chambers (354480, BioCoat). Cell invasive capacity was determined using inserts coated with Matrigel. Briefly, MDA231 and MDABR3 cells were washed by PBS and released from the wells using trypsin. After washing with the serum-free medium twice, cells were resuspended in the serum-free medium. A total of $500 \mu \mathrm{l}$ cell suspension $\left(2 \times 10^{5}\right.$ cells $\left./ \mathrm{ml}\right)$ was seeded in the upper chamber, and cells were plated in L-15 medium (Sangon, Shanghai, China) without FBS. The lower chamber contained $700 \mu \mathrm{l}$ of medium supplemented with $10 \%$ FBS. Cells were incubated in $5 \% \mathrm{CO}_{2}$, at $37^{\circ} \mathrm{C}$. After incubation for $24 \mathrm{~h}$, cells in the upper chamber were removed, and cells on the lower surface were stained with $0.1 \%$ crystal violet. 
2.6. Construction of $B B B$ In Vitro Model. To investigate the function of exosomes, an in vitro model of BBB was established as previously described [16]. In brief, pericytes were plated on the lower surface of the upper chamber and cultured for $24 \mathrm{~h}$. Human BMECs were plated in the upper chamber and cultured to form a uniform monolayer. Astrocytes were cultured in the lower chamber. The BBB in vitro model was successfully constructed when the transepithelial/transendothelial electrical resistance (TEER) value exceeded $150 \mathrm{~cm}^{-2}$.

2.7. Determination of TEER. The barrier function of the BBB model was evaluated by determining its TEER. The resistance values $(O)$ were detected using an ERS-2 Voltohmmeter (Millipore, Billerica, MA). The TEER value was calculated by the resistance per unit area [16] as (measurement resistance value - blank resistance value) $\times 0.33 \mathrm{~cm}^{2}$.

2.8. Permeability Assay. Exosomes were added to the upper chamber of the BBB model and incubated for $24 \mathrm{~h}$. Cells were washed with PBS twice. The upper chamber received $500 \mu \mathrm{l}$ rhodamine $B$ isothiocyanate-dextran solution $(0.1 \mathrm{mg} / \mathrm{ml})$ composed of a FBS-free medium, and the lower chamber contained $1 \mathrm{ml}$ ECM complete medium and culture for $24 \mathrm{~h}$. The cell culture medium was removed from the upper chamber and the lower chamber and added to a 96-well plate in the dark. Measurements were made using a microplate system at $550 \mathrm{~nm}$ and $580 \mathrm{~nm}$, respectively. The permeability rate of each group was calculated as [upper chamber absorbance/ time $(24 \mathrm{~h})^{*}$ lower chamber volume $\left.\left(1 \mathrm{~cm}^{3}\right)\right] /$ (upper chamber absorbance* film area $\left.\left(0.3 \mathrm{~cm}^{2}\right)\right)$.

2.9. Invasion Assay. Invasion of MDA231 breast cancer cells was evaluated by the in vitro model of $\mathrm{BBB}$. The cells were trypsinized and labeled with green fluorescent protein (GFP). Cancer cells were plated with $2 \times 10^{4}$ cells in a serum-free DMEM and the M199 medium containing 10\% serum was used as the chemoattractant in the lower chamber. After $48 \mathrm{~h}$, the noninvading cells were removed and the invading cells labeled with GFP. All assays were performed in triplicate.

2.10. Western Blot. Proteins from exosomes and BMECs were extracted, and the concentration was determined using a BCA assay kit (Pierce Biotechnology, Inc., Rockford, IL, USA). Protein samples with $30 \mu \mathrm{g}$ from each group were separated by $10 \%$ sodium dodecyl sulfate-polyacrylamide gel electrophoresis. The resolved proteins were transferred to polyvinylidene fluoride membranes (Millipore, Bedford, MA, USA). The proteins were blocked in 5\% milk and incubated with primary antibodies against HSP70 (1:2000, \#4873, Cell Signaling Technology, Beverly, MA, USA), CD63 ( $1: 1000$, \#sc-365604, Santa Cruz Biotechnology, Santa Cruz, CA, USA), ZO-1 (1:1000, \#13663, Cell Signaling Technology), claudin-5 (1:1000, \# sc-374221, Santa Cruz Biotechnology), N-cadherin (1:1000, \#13116, Cell Signaling Technology), and glyceraldehyde-3-phosphate dehydrogenase (GAPDH, 1:1000, \#5174, Cell Signaling Technology) overnight at $4^{\circ} \mathrm{C}$. The samples were then incubated with anti-rabbit or anti-mouse horseradish peroxidase-conjugated secondary antibody. Enhanced chemiluminescence reagent (Thermo Fisher Scientific, Waltham, MA, USA) was used to show the protein bands and optical density was assessed via an Image J software.

2.11. Screening for Brain Metastasis-Related IncRNA. The differently expressed lncRNAs between high and low metastatic breast cancer cells were obtained from the data set GSE79540 [10]. The data was downloaded from the GEO database (https://www.ncbi.nlm.nih.gov/geo/query/acc .cgi?acc=GSE79540). The Raw Intensity column was set as the signal value; the seqname was set as ID column. The ID column and the signal value column are deduplicated. Different expressions were analyzed using the DEGseq algorithm, and FDR $<0.05$, log 2Fold Chang $>1$ or $<-1$ was considered significantly different.

2.12. Real-Time PCR. The expressions of lncRNAs were validated by real-time PCR. Total RNA from the exosomes and cells was extracted by TRIzol reagent (Invitrogen). The quantity and quality of the extracted RNA was determined by a NanoDrop 2000 spectrophotometer (Wilmington, DE, USA). The qualified RNA samples with an A260/280 ratio $>1.9$ were used for real-time PCR. A PrimeScript RT kit (Takara Bio, Dalian, China) was used to synthesize complementary DNA (cDNA). Real-time PCR was performed using a SYBR-Green PCR kit (Roche Diagnostics, Indianapolis, IN, USA). PCR was performed on an ABI QuantStudio ${ }^{\text {TM }} 6$ Flex System. U6 was used as the internal reference and the primer sequences used for the PCR are shown in Table 1. The PCR was run at $95^{\circ} \mathrm{C}$ for $10 \mathrm{~min}$ for degeneration, 45 cycles of $95^{\circ} \mathrm{C}$ for $15 \mathrm{sec}$, and $60^{\circ} \mathrm{C}$ for $60 \mathrm{sec}$ and dissociation at $95^{\circ} \mathrm{C}$ for $10 \mathrm{sec}, 60^{\circ} \mathrm{C}$ for $1 \mathrm{~min}$, and $95^{\circ} \mathrm{C}$ for $15 \mathrm{sec}$. The data were analyzed by the $2^{-\Delta \Delta \mathrm{Ct}}$ method. The PCR reactions were all repeated three times.

2.13. Statistical Analysis. SPSS 21.0 (IBM, Chicago, IL, USA) was used to analyze the real-time PCR data. Two-tailed Students' $t$-test was used to compare the differences between two groups. Mean value \pm standard deviation was used to present the experimental data. $P<0.05$ was considered statistically significant.

\section{Results}

3.1. Establishment of Brain Metastatic Cell Lines. To determine the influence of exosomes on the metastatic process of breast cancer, MDA-MB-231-luc-D3H2LN (MDA231) human breast cancer cells were used. The cells have pronounced tumorigenicity and transfer ability and so were used to establish a cell line that readily metastasized to the brain. The MDA231 cells were used for the intracardiac injection into immunodeficiency female mice, and engraftment of cells in the brain was isolated, generating a new high brain metastatic cell line (Figure 1(a)). Brain metastasis was monitored by intraperitoneal injections of luciferin followed by in vivo imaging (Figure 1(b)). After dissociating and expanding brain metastatic tumor in culture, the generated cell group 1 (MDABR1) was selected in the second round in vivo to 
TABle 1: Primer and siRNA sequences.

\begin{tabular}{|c|c|}
\hline Gene symbol & Primer and siRNA sequence \\
\hline \multirow[b]{2}{*}{ KRT19P2 } & $\mathrm{F}^{\prime \prime}$ ACCATTGAGAACGCCAGGATT $3^{\prime}$ \\
\hline & R 5' ACCTCATCCAGCACCCAAAC 3' \\
\hline \multirow{2}{*}{ GS1-600G8.5 } & F 5' CACAGTGAACCGGACAGTCA 3' \\
\hline & R 5' TCAGGCACAATCAGTGGGTC $3^{\prime}$ \\
\hline \multirow{2}{*}{ RP11-176D17.3 } & F $5^{\prime}$ TGCTCATTCATTCATAAATGCTCTT $3{ }^{\prime}$ \\
\hline & R 5' AGACGGGAGACAGGAAGTGA 3' \\
\hline \multirow{2}{*}{ FAM87B } & F 5' TGGGCGTCATTCTTTGTGGT 3' \\
\hline & R 5 $5^{\prime}$ CCATAGATGAGAGCTGGCCG 3' \\
\hline \multirow{2}{*}{ AP000695.4 } & F $5^{\prime}$ TCACAGTGGCCTTGAAGTTCT $3^{\prime}$ \\
\hline & R 5' CGGGTGTTGTTTGTCAGTGC 3' \\
\hline \multirow{2}{*}{$\begin{array}{l}\text { GS1-600G8.5 } \\
\text { siRNA-1 }\end{array}$} & AUUGUUAACCAUUGCAAAGAA \\
\hline & CUUUGCAAUGGUUAACAAUAC \\
\hline \multirow{2}{*}{$\begin{array}{l}\text { GS1-600G8.5 } \\
\text { siRNA-2 }\end{array}$} & UCUACUAGUAUUGUUAACCAU \\
\hline & GGUUAACAAUACUAGUAGAAA \\
\hline \multirow{2}{*}{$\begin{array}{l}\text { GS1-600G8.5 } \\
\text { siRNA-3 }\end{array}$} & UUCUACUAGUAUUGUUAACCA \\
\hline & GUUAACAAUACUAGUAGAAAC \\
\hline \multirow{2}{*}{ siRNA NC } & UUCUCCGAACGUGUCACGUTT \\
\hline & ACGUGACACGUUCGGAGAATT \\
\hline
\end{tabular}

produce brain metastatic cell group 2 cells (MDABR2). The MDABR2 cells were subjected to a third round of in vivo selection, yielding MDABR3 cell populations that displayed significantly increased brain metastasis. Following ventricle injection, MDA231 cells yielded brain metastases in three of $10(30 \%)$ mice with brain metastases, whereas MDABR3 cells yielded brain metastases in eight of 10 injected mice (80\%). In vitro, the Transwell assay demonstrated that the migratory and invasive potential of MDABR3 cells was significantly increased compared to that of MDA231 cells (Figure 1(c)).

3.2. BBB In Vitro Model. To investigate the mechanism by which breast cancer cells pass through the $\mathrm{BBB}$, we establish an in vitro $\mathrm{BBB}$ culture system. In vivo, the $\mathrm{BBB}$ mainly consists of three types of cells-BMECs, pericytes, and astrocytes-which cooperate with each other to guarantee the function of $\mathrm{BBB}$. Therefore, we constructed an in vitro BBB model system in which BMECs were seeded on the upper surface of the upper compartment, pericytes were inoculated on the lower surface of the upper compartment, and astrocytes were cultured in the lower compartment (Figure 2(a)). TEER was used to measure the formation of tight connections between endothelial cells in brain microvessels, which indicated the integrity of the $\mathrm{BBB}$. The TEER of the $\mathrm{BBB}$ model was high enough to serve as a model of the $\mathrm{BBB}$ in vivo (Figure 2(b)). Moreover, the permeability experiment showed that the permeability of the BBB system was very low on days 4 and 5 , consistent with the results of
TEER (Figure 2(c)). To investigate how exosomes mediated the metastasis of breast cancer cells through the BBB, we incubated the BBB model with exosomes for $24 \mathrm{~h}$, and GFP-labeled breast cancer cells were added to the upper chamber of the BBB model (Figure 2(d)). The effect of exosomes on the BBB was evaluated by counting the GFPlabeled breast cancer cells that successfully crossed the BBB system.

3.3. Identification of Exosomes Derived from Breast Cancer Cells. To evaluate the exosomal roles in brain metastases, we isolated the exosomes from the high brain metastatic MDABR3 cells and low brain metastatic MDA231 cells. TEM and western blot were used to identify the isolated exosomes. TEM revealed that the exosomes were 100 to $150 \mathrm{~nm}$ in size with a complete membrane structure (Figure 3(a)). Western blot showed that the exosomes significantly expressed the exosomal markers CD63 and HSP70 (Figure 3(b)). The results suggested the successful isolation of exosomes from breast cancer cells.

3.4. Exosomes Derived from High Metastatic Cancer Cells Destroy the $B B B$. To investigate the influence of exosomes on the BBB system, exosomes were labeled with PKH26 and added to the BBB model. As shown in Figure 3(c), exosomes from all the breast cancer cells, including MDABR3 and MDA231, were incorporated into BMECs. Importantly, the changes in TEER of the BBB model induced by MDABR3 exosomes were significantly higher than the changes induced by MDA231 exosomes (Figure 3(d)). MDABR3 exosomes significantly increased the relative permeability of the BBB model compared to the blank group (Figure 3(e)). Moreover, the permeability of BBB model treated with MDABR3 exosomes was significantly higher than that treated with MDA231 exosomes (Figure 3(e)). Furthermore, to clarify whether the exosomes were sufficient to allow the migration of cancer cells to the parenchymal side of the brain, we determined the extravasation of low metastatic cancer cells posttreating the in vitro $\mathrm{BBB}$ model with exosomes derived from the MDA231 or MDABR3 cells. As shown in Figure 3(f), low metastatic MDA231 cells could not pass through $\mathrm{BBB}$ without the pretreatment of exosomes, whereas the low metastatic MDA231 cells successfully infiltrated to the abluminal side after treating the BBB system with exosomes derived from high metastatic MDABR3 cells. In contrast, exosomes derived from MDA231 cells failed to enhance the migration of low metastatic MDA231 cells through $\mathrm{BBB}$ compared with exosomes derived from MDABR3 cells. Taken together, these data indicate that the exosomes secreted from high metastatic breast cancer cells destroy the $\mathrm{BBB}$ permeability and thus promote the passage of cancer cells across the BBB.

3.5. Exosomes Destroy BBB by Transferring IncRNA GS1600G8.5. To explore the underlying mechanism by which exosomes from high metastatic cells mediated the reconstruction of $\mathrm{BBB}$, we analyzed the lncRNAs differently expressed between high and low metastatic breast cancer cells. From the data set GSE79540 [10], we obtained 1,583 


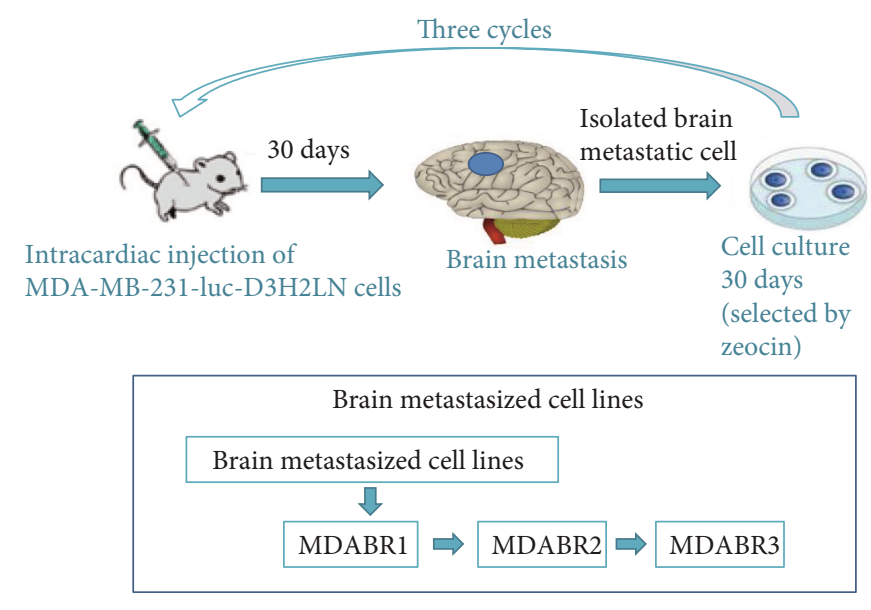

(a)

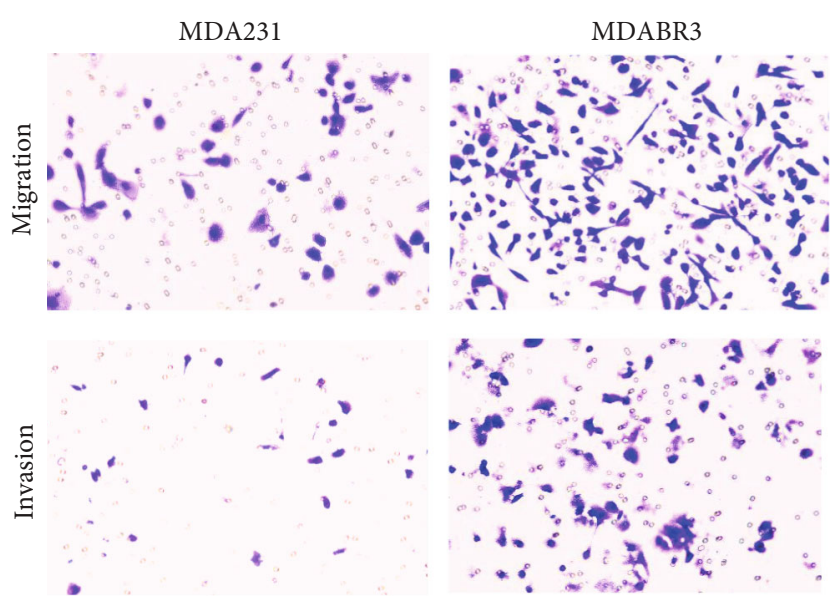

(c)

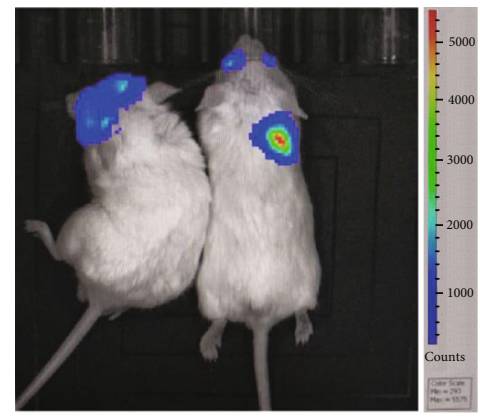

30 days after injection

(b)

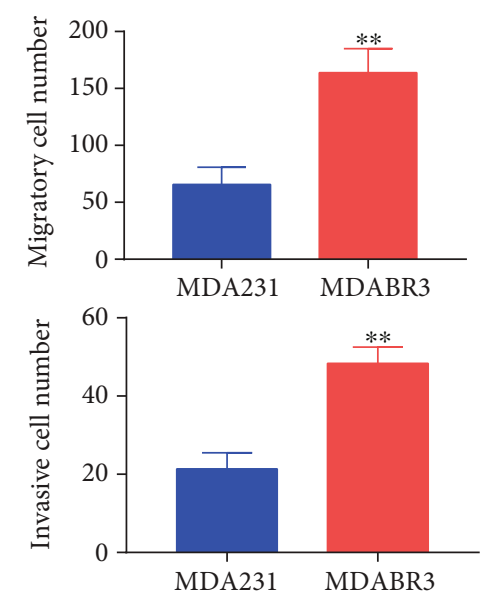

Figure 1: Establishment of breast cancer cell lines with high brain metastatic capacity. (a) Schematic diagram shows the protocol for the in vivo selected brain metastatic cells. Breast cancer cells (MDA-MB-231-luc-D3H2LN, MDA231) were injected into the left ventricle of 6-8 week old female wild-type BALB/c mice. After 30 days, biological imaging was performed to observe brain metastases. Cells that had metastasized in the brains of mice were isolated and defined as MDABR1. Half of the MDABR1 cells were frozen, and half were reinjected into mice. After 30 days, biological imaging was performed to observe the brain metastasis, and metastasized cells were isolated and defined as MDABR2. MDABR2 cells were subjected to a third selection to generate MDABR3 $(N=10)$. (b) Bioluminescent images of MDABR3 brain metastases in mice. (c) In vitro Transwell assay showing the migration and invasion capacities of MDA231 and MDABR3 cells $\left(N=3\right.$, $t$-test, $\left.{ }^{* *} P<0.01\right)$.

differently expressed lncRNAs, including 654 up- and 929 downregulated lncRNAs, in brain metastatic breast cancer cells compared to parental cells (Figure 4(a)). We selected the top five upregulated lncRNAs and verified their expressions in our high brain metastatic cell lines. Consistently, the expressions of KRT19P2, GS1-600G8.5, RP11176D17.3, and AP000695.4 were significantly increased in the high brain metastatic MDABR3 cells compared to the low brain metastatic MDA231 cells (Figure 4(b)). Interestingly, only KRT19P2 and GS1-600G8.5 showed upexpression in the exosomes derived from high brain metastatic MDABR3 cells compared to the exosomes from low metastatic MDA231 cells (Figure 4(c)). Moreover, GS1-600G8.5 displayed a higher fold-change compared to KRT19P2. Therefore, we further investigated the effect of GS1-600G8.5 on BBB.
After transfection with GS1-600G8.5 small interfering RNAs (siRNAs), exosome was isolated from the siRNAtransfected MDABR3 cells and generated a GS1-600G8.5deficient exosome (Figure 4(d)). Moreover, siRNA-2 showed the highest interference effect and was selected for further investigation. As expected, MDABR3 exosomes remarkably increased the changes in the TEER of the BBB model, while the deficiency of GS1-600G8.5 abrogated the effect of MDABR3 exosomes (Figure 4(e)). Similarly, MDABR3 exosomes significantly increased the permeability of the $\mathrm{BBB}$ model, whereas silencing of GS1-600G8.5 in MDABR3 exosomes dramatically restored the $\mathrm{BBB}$ permeability to the normal level (Figure 4(f)). The low brain metastatic MDA231 cells successfully infiltrated the $\mathrm{BBB}$ system by addition with exosomes from NC-transfected MDABR3 cells. However, the exosomes from GS1-600G8.5 siRNA-transfected 


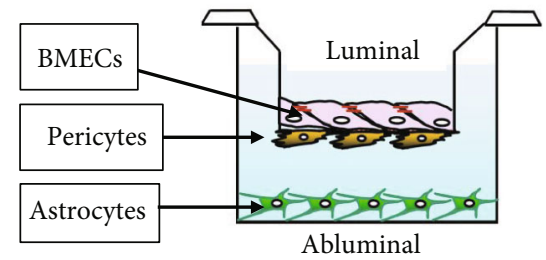

(a)

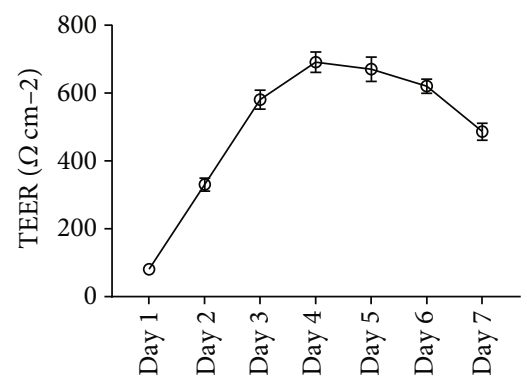

(b)

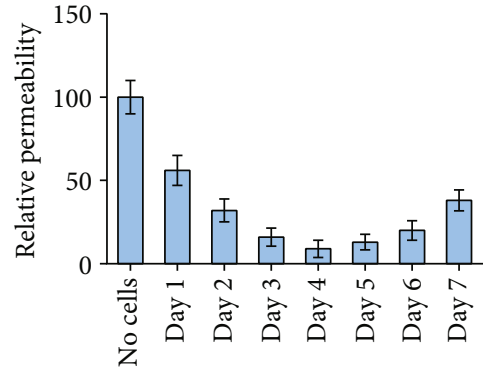

(c)
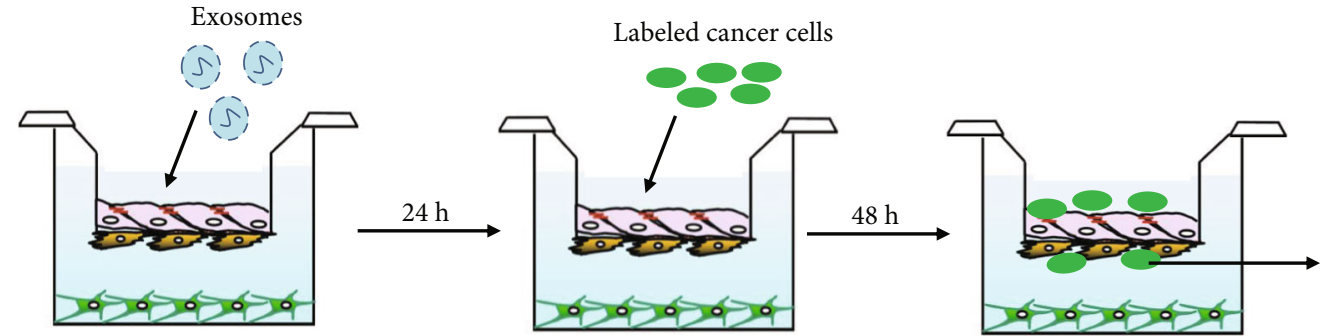

Count the invaded

(d)

FIGURE 2: Establishment of the in vitro BBB system. (a) Schematic diagram showing the construction of the in vitro BBB model, using human brain microvascular endothelium cell (BMEC), brain pericytes, and astrocytes. (b) Transepithelial/transendothelial electrical resistance (TEER) detection in the BBB model. (c) Permeability of the BBB model. The permeability of a cell-free system was used as the control. (d) Schematic diagram displayed the methods for detecting exosome effects on invasion of breast cancer cells through the BBB model.

MDABR3 cells did not facilitate the invasion of low brain metastatic MDA231 cells through the BBB (Figure 4(g)). To further study the mechanism of GS1-600G8.5, we detected the expression of tight junction proteins in BMECs. The expressions of ZO-1, claudin-5, and N-cadherin proteins were significantly decreased in the BMECs treated with MDABR3 exosomes compared to that without treatment (Figure 4(h)). With GS1-600G8.5 deficiency in the exosome, the expressions of ZO-1, Claudin-5, and N-cadherin proteins were increased in the BMECs. These results suggested that exosomes containing lncRNA GS1-600G8.5 may destroy $\mathrm{BBB}$ by the tight junction proteins.

\section{Discussion}

Emerging evidences have revealed that exosomes play a crucial role in the metastatic process of cancer [17]. lncRNAs have been shown to be involved in the occurrence and progression of cancer via various mechanisms [18]. However, whether the exosomes modulate the brain metastasis of cancer by carrying lncRNAs remains unknown. In the present study, we successfully established a breast cancer cell line that readily metastasized to the brain. Exosomes derived from these metastatic breast cancer cells could destroy the BBB and thus promote passage of the cancer cells across the BBB. The exosome-facilitated mechanism involved lncRNA GS1-600G8.5.

In the tumor microenvironment, exosomes mediate cancer metastasis mainly by regulating premetastatic niche formation, vascular permeability, macrophage polarization, and tumor-related fibroblasts and other stromal cells.
Recently, the role of exosomes in brain metastasis has also attracted attention. Astrocyte-derived exosomes can transfer miRNA-142-3p to lung adenocarcinoma cells and inhibit brain metastasis by suppressing fibroblast growth factor receptor 2 activation [10]. Astrocyte exosomal miR-19a reversibly downregulates the expression of PTEN in breast cancer cells, promoting brain metastasis of breast cancer [14]. Breast cancer cell-derived miR-122 inhibits the glucose uptake of brain astrocytes by downregulating the glycolytic enzyme pyruvate kinase, which promotes brain metastasis [19]. IncRNA XIST knockout promotes the secretion of exosomal miRNA-503 from breast cancer cells, which promotes brain metastasis by mediating microglia cell polarization [20]. The key process for the metastasis of cancer cells to brain is crossing the BBB. How this is done has been unclear. A previous study showed that breast cancer cells release extracellular vesicles containing miR181c to promote brain metastasis by disrupting the BBB [16]. Similarly, our study revealed that IncRNA GS1-600G8.5 containing exosomes derived from breast cancer cells that were prone to undergo brain metastasis disrupted the BBB.

Compared with exosomal miRNA, the role of exosomal lncRNA has been poorly studied. Recently, several exosomal lncRNAs were revealed to be involved in breast cancer. Breast cancer exosomes promote cell proliferation by transferring IncRNA MALAT [21]. Exosome-mediated delivery of lncRNA SNHG14 induces breast cancer chemoresistance to trastuzumab by regulating apoptosis regulators [22]. HOTAIR lncRNA is expressed in exosomes derived from breast cancer patients and is associated with ErbB2/HER2 positivity [23]. Moreover, exosomal lncRNA has been shown 


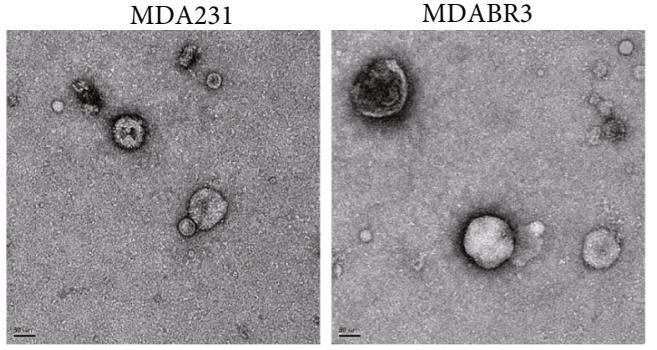

(a)
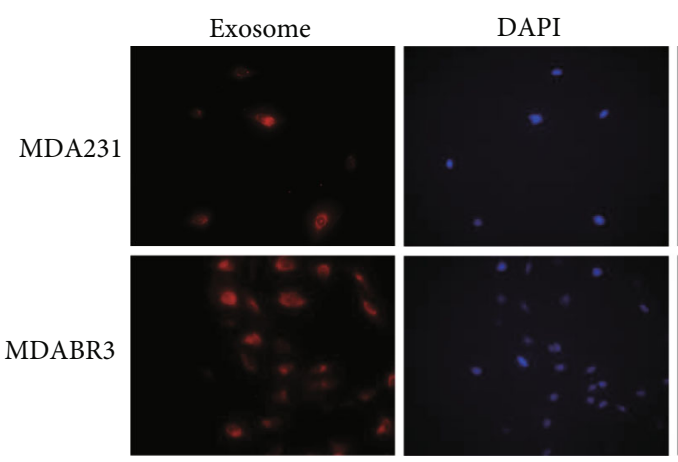

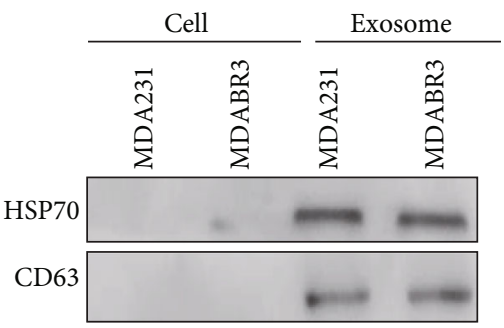

(b) (c)

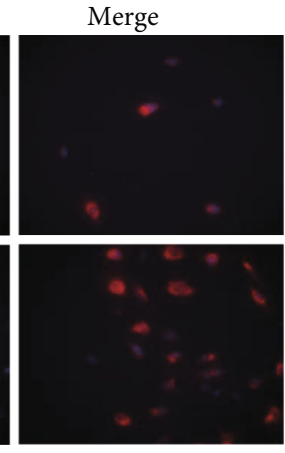

MDA231 cells

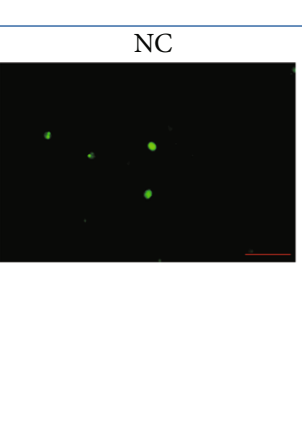

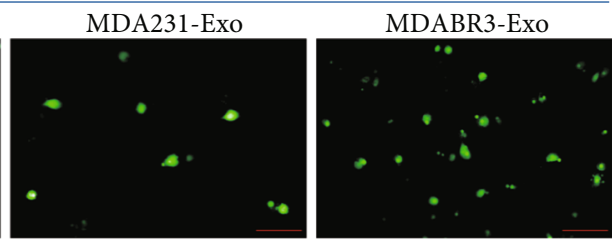

(f)

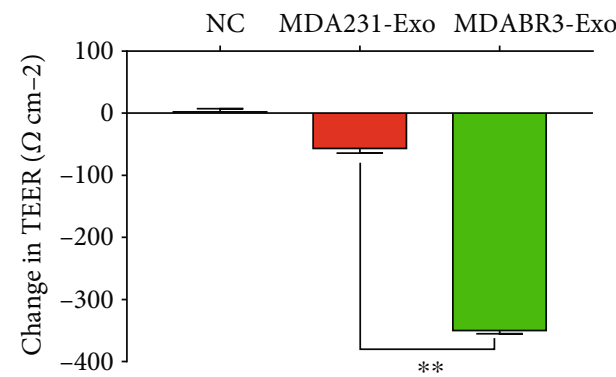

(d)

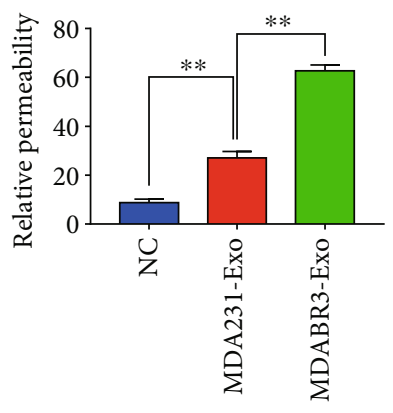

(e)

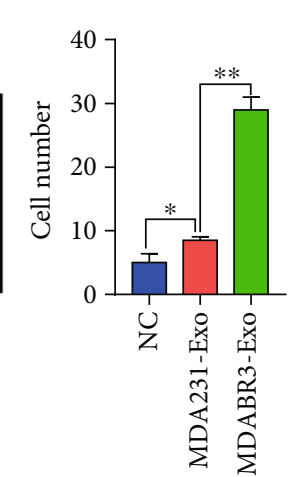

Figure 3: Exosomes derived from high brain metastatic cells destroys BBB permeability. (a) Transmission electron microscopy appearance of exosomes. (b) Western blot measurement of exosome markers in cells and exosomes. (c) Exosomes derived from MDA231 low metastatic breast cancer cells and MDABR3 high brain metastatic breast cancer cells were labeled with PKH26 and added to the BBB model. Representative pictures of BMECs internalizing the exosomes (magnification $\times 200$ ). (d) Exosomes were added to the BBB system and the TEER value was monitored after $24 \mathrm{~h}$. NC: negative control (BBB system without exosome treatment). (e) Permeability was detected after exosomes incubated BBB system for $24 \mathrm{~h}$. (f) Exosomes were added to the BBB model and incubated for $24 \mathrm{~h}, \mathrm{MDA} 231$ (PFG-labeled) cells were added to the BBB model, and the number of breast cancer cells crossing the BBB were counted after $48 \mathrm{~h}$. (Magnification $\times 200$, one-way ANOVA, $\left.{ }^{*} P<0.05,{ }^{* *} P<0.01\right)$.

to play important roles in the absence of brain metastasis [24]. However, the role of IncRNA shuttled by exosomes in the brain metastasis or breast cancer metastasis has not been reported. In this study, for the first time, we used an in vitro system to reveal that high brain metastatic cancer cell-derived exosomal lncRNA GS1-600G8.5 can disrupt the $\mathrm{BBB}$, which promotes the passage of breast cancer cells through the BBB. This data indicates that exosomal lncRNA GS1-600G8.5 might promote brain metastasis of breast cancer. In vivo data from an animal model are needed for verification. Furthermore, exosomal lncRNA GS1-600G8 decreased the expression of tight junction proteins, suggesting that exosomal lncRNA GS1-600G8 might disrupt the $\mathrm{BBB}$ by targeting tight junction proteins. The mechanism of exosomal lncRNA GS1-600G8.5 remains to be explored in studies that are planned.

Exosomes have been shown to carry various molecules and drugs across the $\mathrm{BB}$ in vivo. Exosomes loaded with miR-193b-3p can pass through the BBB and efficiently deliver miR-193b-3p into the hemorrhage region of the brain, which alleviates neuroinflammation in the brains of mice with subarachnoid hemorrhage [25]. Yang et al. showed exosomes loaded with anticancer can across the blood-brain barrier in vivo, which suppressed tumor growth [26]. Exosome-delivered GAPDH-siRNA can pass through the $\mathrm{BBB}$ and specifically enter neurons, microglia, 


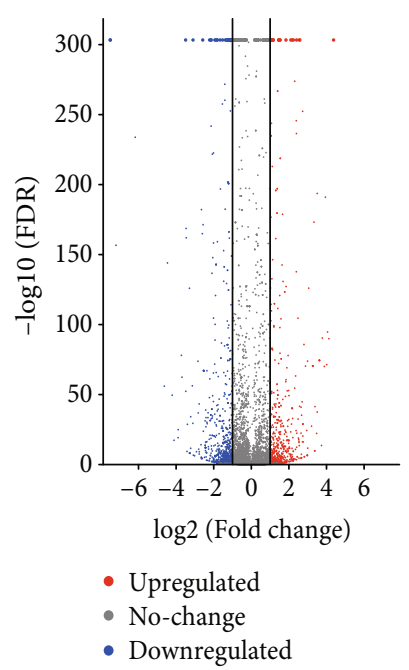

(a)

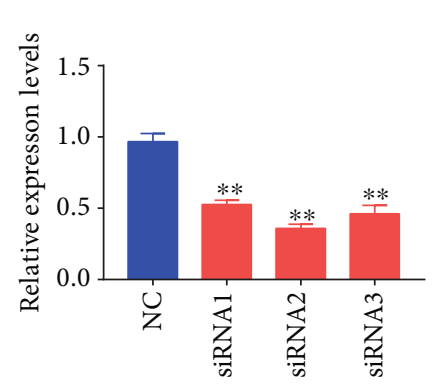

(d)

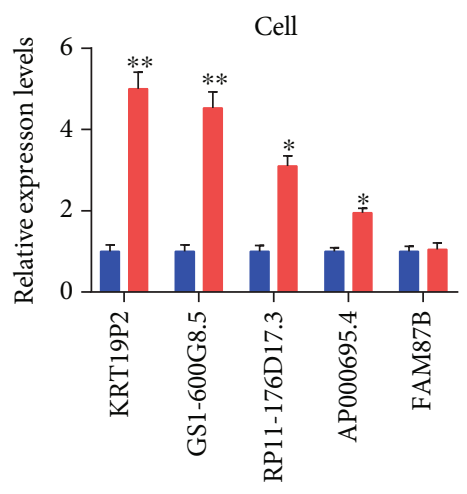

MDA231

MDABR3

(b)

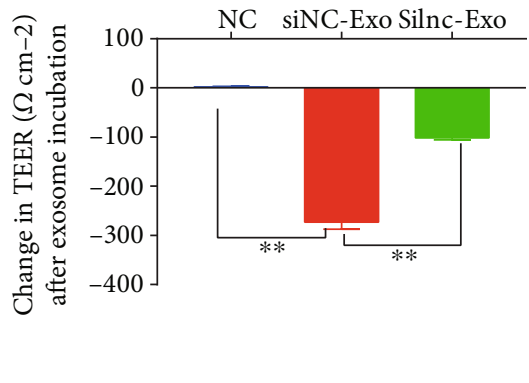

(e)

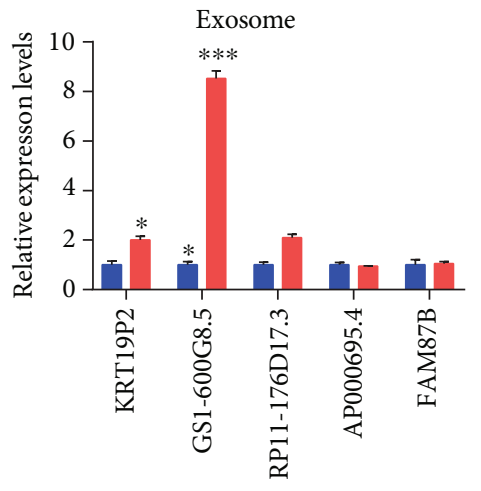

MDA231-Exo

MDABR3-Exo

(c)

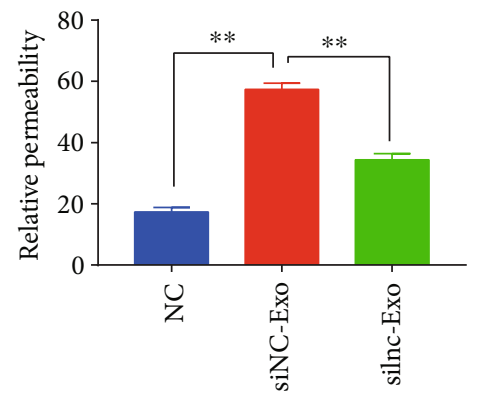

(f)

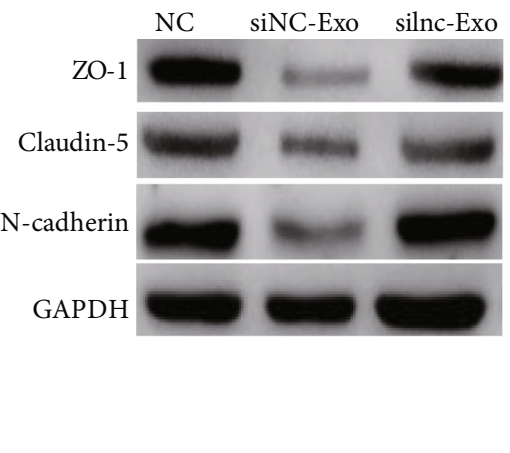

(h)

(g)

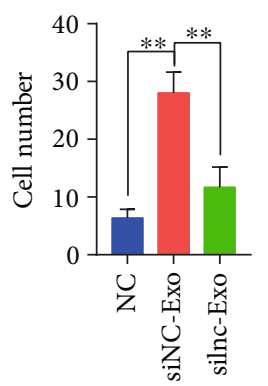

FIGURE 4: Exosomal lncRNA GS1-600G8.5 promotes BBB passage of breast cancer cells. (a) Volcano plot of differentially expressed lncRNAs between brain metastatic and nonbrain metastatic breast cancer cells. Data were obtained from the GSE79540 database [10]. The red dots represent upregulated lncRNAs and the blue dots represent downregulated lncRNAs in the brain metastatic breast cancer cells compared to the nonbrain metastatic breast cancer cells. (b) The top five upregulated lncRNAs were verified by real-time PCR, in MDABR3 high brain metastatic breast cancer cells and MDA231 parental cells $(N=3, t$-test). (c) The top five upregulated lncRNAs were verified by realtime PCR in exosomes derived from MDABR3 and parental MDA231 cells ( $N=3, t$-test). (d) The interference effect of GS1-600G8.5 was verified by real-time PCR in exosomes derived from MDABR3 cells transfected with siRNA. The siRNA-2 with the best interference effect was used for further study. One-way ANOVA. (e) GS1-600G8.5-deficient MDABR3 exosomes and control exosomes were added to the BBB system, and the change in the TEER value was detected after $24 \mathrm{~h}$. One-way ANOVA comparison was done. NC: BBB system without any treatment; siNC-Exo: BBB system treated with exosomes derived from MDABR3 cells that were transfected with siRNA NC; silnc-Exo: BBB system treated with exosomes derived from MDABR3 cells that were transfected with siRNA GS1-600G8.5. (f) Permeability was detected in the BBB system. One-way ANOVA comparison was used. (g) BBB system was treated with exosomes for 24h, MDA231 (PFG-labeled) cells were added to BBB model, and the number of MDA231 cells crossing the BBB was counted after 48 h. Magnification $\times 200$. One-way ANOVA. (h) Western blot was used to detect the expressions of tight junction and adhesive proteins in the BMECs treated with GS1600G8.5-deprived exosomes and control exosomes. One-way ANOVA comparison was used; ${ }^{*} P<0.05,{ }^{* *} P<0.01$, and ${ }^{* * *} P<0.001$. 
and oligodendrocytes in the brain [27]. Exosomes derived from breast cancer cells breach the intact $\mathrm{BBB}$ and are taken up by various cells including the brain parenchyma, indicating their capacity to go beyond the $\mathrm{BBB}$ in vivo [28]. However, the ability of lncRNA containing exosomes to cross the $\mathrm{BBB}$ and the mechanisms involved in this process remain unknown. In the present study, we showed that the GS1-600G8.5 containing exosomes was internalized by $\mathrm{BBB}$ in vitro. Based on the above evidences that exosomes carrying various molecules can cross the BBB in brain, we speculate that lncRNAs carried by exosomes might also cross the $\mathrm{BBB}$ in vivo. Exosomes have been recommended as novel promising therapeutics and drug delivery vehicles [29]. Our study showed that exosomal lncRNA GS1-600G8.5 can overcome the BBB and contribute to the progression of brain metastasis in vitro, indicating that exosomal lncRNA might act as promising therapeutic targets for brain metastasis in vivo.

In conclusion, IncRNA GS1-600G8.5 was highly expressed in exosomes derived from breast cancer cells that readily metastasized to the brain, compared to the lncRNA in exosomes from low metastatic cells. Moreover, exosomal lncRNA GS1-600G8.5 disrupted the BBB and promoted the passage of breast cancer cells across the BBB, perhaps by targeting tight junction proteins. This data provides new insights into the roles of exosomal lncRNAs in cancer metastasis to the brain.

\section{Data Availability}

The data sets used and/or analyzed during the current study are available from the corresponding author on reasonable request.

\section{Conflicts of Interest}

The authors declare that they have no competing interests, and all authors should confirm its accuracy.

\section{Acknowledgments}

This study was supported by the National Natural Science Foundation of China Youth Program (81902530).

\section{References}

[1] F. Bray, J. Ferlay, I. Soerjomataram, R. L. Siegel, L. A. Torre, and A. Jemal, "Global cancer statistics 2018: GLOBOCAN estimates of incidence and mortality worldwide for 36 cancers in 185 countries," CA: a Cancer Journal for Clinicians, vol. 68, no. 6, pp. 394-424, 2018.

[2] D. P. Kodack, V. Askoxylakis, G. B. Ferraro, D. Fukumura, and R. K. Jain, "Emerging strategies for treating brain metastases from breast cancer," Cancer Cell, vol. 27, no. 2, pp. 163-175, 2015.

[3] I. Dagogo-Jack, C. M. Gill, D. P. Cahill, S. Santagata, and P. K. Brastianos, "Treatment of brain metastases in the modern genomic era," Pharmacology \& Therapeutics, vol. 170, pp. 64-72, 2017.

[4] R. Di Lorenzo and M. S. Ahluwalia, "Targeted therapy of brain metastases: latest evidence and clinical implications,"
Therapeutic Advances in Medical Oncology, vol. 9, no. 12, pp. 781-796, 2017.

[5] A. M. Schmitt and H. Y. Chang, "Long noncoding RNAs in cancer pathways," Cancer Cell, vol. 29, no. 4, pp. 452-463, 2016.

[6] J. Beermann, M. T. Piccoli, J. Viereck, and T. Thum, "Noncoding RNAs in development and disease: background, mechanisms, and therapeutic approaches," Physiological Reviews, vol. 96, no. 4, pp. 1297-1325, 2016.

[7] Y. Lian, F. Xiong, L. Yang et al., "Long noncoding RNA AFAP1-AS1 acts as a competing endogenous RNA of miR423-5p to facilitate nasopharyngeal carcinoma metastasis through regulating the Rho/Rac pathway," Journal of Experimental \& Clinical Cancer Research, vol. 37, no. 1, p. 253, 2018.

[8] J. Kim, H. L. Piao, B. J. Kim et al., "Long noncoding RNA MALAT1 suppresses breast cancer metastasis," Nature Genetics, vol. 50, no. 12, pp. 1705-1715, 2018.

[9] B. S. Tan, M. C. Yang, S. Singh et al., "LncRNA NORAD is repressed by the YAP pathway and suppresses lung and breast cancer metastasis by sequestering S100P," Oncogene, vol. 38, no. 28, pp. 5612-5626, 2019.

[10] S. Wang, K. Liang, Q. Hu et al., "JAK2-binding long noncoding RNA promotes breast cancer brain metastasis," The Journal of Clinical Investigation, vol. 127, no. 12, pp. 4498-4515, 2017.

[11] G. van Niel, G. D'Angelo, and G. Raposo, "Shedding light on the cell biology of extracellular vesicles," Nature Reviews. Molecular Cell Biology, vol. 19, no. 4, pp. 213-228, 2018.

[12] H. Peinado, M. Alečković, S. Lavotshkin et al., "Melanoma exosomes educate bone marrow progenitor cells toward a pro- metastatic phenotype through MET," Nature Medicine, vol. 18, no. 6, pp. 883-891, 2012.

[13] J. H. Fang, Z. J. Zhang, L. R. Shang et al., "Hepatoma cell-secreted exosomal microRNA-103 increases vascular permeability and promotes metastasis by targeting junction proteins," Hepatology, vol. 68, no. 4, pp. 1459-1475, 2018.

[14] L. Zhang, S. Zhang, J. Yao et al., "Microenvironment-induced PTEN loss by exosomal microRNA primes brain metastasis outgrowth," Nature, vol. 527, no. 7576, pp. 100-104, 2015.

[15] P. D. Bos, X. H.-F. Zhang, C. Nadal et al., "Genes that mediate breast cancer metastasis to the brain," Nature, vol. 459, no. 7249, pp. 1005-1009, 2009.

[16] N. Tominaga, N. Kosaka, M. Ono et al., "Brain metastatic cancer cells release microRNA-181c-containing extracellular vesicles capable of destructing blood-brain barrier," Nature Communications, vol. 6, no. 1, p. 6716, 2015.

[17] R. Xu, A. Rai, M. Chen, W. Suwakulsiri, D. W. Greening, and R. J. Simpson, "Extracellular vesicles in cancer - implications for future improvements in cancer care," Nature Reviews. Clinical Oncology, vol. 15, no. 10, pp. 617-638, 2018.

[18] F. Kopp and J. T. Mendell, "Functional classification and experimental dissection of long noncoding RNAs," Cell, vol. 172, no. 3, pp. 393-407, 2018.

[19] M. Y. Fong, W. Zhou, L. Liu et al., "Breast-cancer-secreted miR-122 reprograms glucose metabolism in premetastatic niche to promote metastasis," Nature Cell Biology, vol. 17, no. 2, pp. 183-194, 2015.

[20] F. Xing, Y. Liu, S. Y. Wu et al., "Loss of XIST in breast cancer activates MSN-c-met and reprograms microglia via exosomal miRNA to promote brain metastasis," Cancer Research, vol. 78, no. 15, pp. 4316-4330, 2018. 
[21] P. Zhang, H. Zhou, K. Lu, Y. Lu, Y. Wang, and T. Feng, "Exosome-mediated delivery of MALAT1 induces cell proliferation in breast cancer," OncoTargets and Therapy, vol. 11, pp. 291299, 2018.

[22] H. Dong, W. Wang, R. Chen et al., "Exosome-mediated transfer of lncRNA-SNHG14 promotes trastuzumab chemoresistance in breast cancer," International Journal of Oncology, vol. 53, no. 3, pp. 1013-1026, 2018.

[23] Y. L. Wang, L. C. Liu, Y. Hung et al., "Long non-coding RNA HOTAIR in circulatory exosomes is correlated with ErbB2/HER2 positivity in breast cancer," Breast, vol. 46, pp. 64-69, 2019.

[24] X. Yang, J. Yang, P. Lei, and T. Wen, "LncRNA MALAT1 shuttled by bone marrow-derived mesenchymal stem cells-secreted exosomes alleviates osteoporosis through mediating microRNA-34c/SATB2 axis," Aging, vol. 11, no. 20, pp. 8777-8791, 2019.

[25] N. Lai, D. Wu, T. Liang et al., "Systemic exosomal miR-193b$3 p$ delivery attenuates neuroinflammation in early brain injury after subarachnoid hemorrhage in mice," Journal of Neuroinflammation, vol. 17, no. 1, p. 74, 2020.

[26] T. Yang, P. Martin, B. Fogarty et al., "Exosome delivered anticancer drugs across the blood-brain barrier for brain cancer therapy in Danio rerio," Pharmaceutical Research, vol. 32, no. 6, pp. 2003-2014, 2015.

[27] L. Alvarez-Erviti, Y. Seow, H. Yin, C. Betts, S. Lakhal, and M. J. Wood, "Delivery of siRNA to the mouse brain by systemic injection of targeted exosomes," Nature Biotechnology, vol. 29, no. 4, pp. 341-345, 2011.

[28] G. Morad, C. V. Carman, E. J. Hagedorn et al., "Tumorderived extracellular vesicles breach the intact blood-brain BarrierviaTranscytosis," ACS Nano, vol. 13, no. 12, pp. 13853-13865, 2019.

[29] J. J. Mulvihill, E. M. Cunnane, A. M. Ross, J. T. Duskey, G. Tosi, and A. M. Grabrucker, "Drug delivery across the blood-brain barrier: recent advances in the use of nanocarriers," Nanomedicine, vol. 15, no. 2, pp. 205-214, 2020. 